\title{
ASPECTOS SOCIOECONÔMICOS E EVENTOS DE QUEDA ENTRE IDOSOS ATENDIDOS NO SISTEMA PÚBLICO DE SAÚDE
}

\section{Socioeconomic aspects and falls in older adults served by the public health system \\ Aspectos socioeconómicos y eventos de caídas en mayores asistidos por el sistema público de salud}

\author{
Ériks Oliveira Silva iD \\ Universidade Federal de Uberlândia - UFU - Ituiutaba (MG) - Brasil
}

\author{
Alexandre Azenha Alves de Rezende \\ Universidade Federal de Uberlândia - UFU - Ituiutaba (MG) - Brasil \\ Luciana Karen Calábria ii \\ Universidade Federal de Uberlândia - UFU - Ituiutaba (MG) - Brasil
}

\section{RESUMO}

Objetivo: Apresentar os aspectos socioeconômicos e a ocorrência de eventos de queda entre idosos atendidos no sistema municipal de saúde. Métodos: Estudo descritivo e transversal realizado com 154 idosos, de ambos os sexos (feminino, $n=88$; masculino, $n=66$ ), atendidos nas unidades de saúde do município de Ituiutaba, Minas Gerais, em 2018. Realizou-se a coleta dos dados a partir da aplicação de questionário semiestruturado contendo questões socioeconômicas (escolaridade, ocupação, origem, renda e situação conjugal) e de condição clínica, incluindo eventos de queda. Analisaram-se as variáveis por estatística descritiva. Resultados: Dos idosos, $80,5 \%(n=124)$ era de origem rural, com maior prevalência de mulheres $(57,1 \%$; $n=68)$. Sobre as quedas e seus aspectos relacionados, $37,7 \%(n=58)$ dos idosos afirmaram ter sofrido algum evento nos últimos 12 meses, sendo mais prevalente entre aqueles com idade entre 70 e 79 anos (55,2\%; $n=32)$, do sexo feminino $(67,2 \%$; $n=39)$. Em decorrência da queda, 32,7\% $(n=19)$ apresentaram fratura e, entre aqueles que caíram, $41,4 \%(n=24)$ relataram que a residência apresentava riscos para novos eventos de queda. Conclusão: Os eventos de queda autorreferidos pelos idosos investigados associam-se aos fatores inerentes do envelhecimento biológico, com acentuada feminização do envelhecimento, mas também aos fatores extrínsecos associados ao ambiente onde vivem.

Descritores: Envelhecimento; Vulnerabilidade Social; Acidentes por Quedas; Saúde do Idoso; Promoção da Saúde.

\begin{abstract}
Objective: To present the socioeconomic aspects and the occurrence of falls in older patients served by the municipal health system. Methods: This descriptive cross-sectional study was conducted with 154 older adults of both sexes (women, $n=88 ;$ men, $n=66)$ served at health care centers in the municipality of Ituiutaba, Minas Gerais, in 2018. Data were collected using a semistructured questionnaire that addressed socioeconomic issues (education, occupation, origin, income and marital status) and clinical conditions, including occurrence of falls. The variables were analyzed using descriptive statistics. Results: In all, $80.5 \%$ $(n=124)$ of the older adults were from rural areas and there was a higher prevalence of women (57.1\%; $n=68)$. Regarding falls and related aspects, $37.7 \%(n=58)$ of the older adults reported at least one fall in the past 12 months, with a higher prevalence among those aged $70-79$ years (55.2\%; $n=32$ ) and women (67.2\%; $n=39)$. Fracture resulting from fall occurred in $32.7 \%(n=19)$ of the older adults and $41.4 \%(n=24)$ of those who fell said the house presented risks for new falls. Conclusion: Falls self-reported by the older adults analyzed were associated with factors inherent to biological aging and there was a marked feminization of aging. However, they were also associated with extrinsic factors related to the environment where they live.
\end{abstract}

Descriptors: Aging; Social Vulnerability; Accidental Falls; Health of the Elderly; Health Promotion. 


\section{RESUMEN}

Objetivo: Apresentar os aspectos socioeconômicos e a ocorrência de eventos de queda entre idosos atendidos no sistema municipal de saúde. Métodos: Estudo descritivo e transversal realizado com 154 idosos, de ambos os sexos (feminino, $n=88$; masculino, $n=66$ ), atendidos nas unidades de saúde do município de Ituiutaba, Minas Gerais, em 2018. Realizou-se a coleta dos dados a partir da aplicação de questionário semiestruturado contendo questões socioeconômicas (escolaridade, ocupação, origem, renda e situação conjugal) e de condição clínica, incluindo eventos de queda. Analisaram-se as variáveis por estatística descritiva. Resultados: Dos idosos, $80,5 \%(n=124)$ era de origem rural, com maior prevalência de mulheres $(57,1 \%$; $n=68)$. Sobre as quedas e seus aspectos relacionados, 37,7\% $(n=58)$ dos idosos afirmaram ter sofrido algum evento nos últimos 12 meses, sendo mais prevalente entre aqueles com idade entre 70 e 79 anos (55,2\%; $n=32)$, do sexo feminino $(67,2 \%$; $n=39)$. Em decorrência da queda, 32,7\% (n=19) apresentaram fratura e, entre aqueles que caíram, 41,4\% (n=24) relataram que a residência apresentava riscos para novos eventos de queda. Conclusión: Os eventos de queda autorreferidos pelos idosos investigados associam-se aos fatores inerentes do envelhecimento biológico, com acentuada feminização do envelhecimento, mas também aos fatores extrínsecos associados ao ambiente onde vivem.

Descriptores: Envelhecimento; Vulnerabilidade Social; Acidentes por Quedas; Saúde do Idoso; Promoção da Saúde.

\section{INTRODUÇÃO}

O envelhecimento populacional é marcado por algumas modificações na dinâmica populacional, tanto em países desenvolvidos quanto em desenvolvimento, além de um conjunto de fatores caracterizados por alterações biológicas ao longo da vida ${ }^{(1)}$. Com o processo de transição demográfica (1940 a 2017), a expectativa de vida da população aumentou $30,5 \%$, projetando uma média de 76 anos ${ }^{(2)}$, refletindo a atual constituição da população de 29,6 milhões de idosos no Brasil( ${ }^{(3)}$.

Concomitantemente a essas alterações na estrutura etária do país, devidas ao crescimento de longevos, o acúmulo de doenças relacionadas ao envelhecimento é mais evidenciado, intensificando a demanda por atendimento em serviços de saúde e elevando exponencialmente os gastos no Sistema Único de Saúde (SUS) com internações hospitalares e cuidados médicos ${ }^{(4,5)}$. Só no ano de 2013 há registros de 93.312 internações no SUS em decorrência de queda entre idosos acima de 60 anos, triplicando após os 65 anos, sem contar os gastos no tratamento de fraturas ${ }^{(6,7)}$.

Na busca para amenizar os riscos e os agravos em saúde por meio da atenção básica, o SUS tem, desde a sua criação, consolidado um sistema de atendimento destinado à indissociabilidade de seus princípios, como a integralidade e a universalidade, e, diante das mudanças na população, novos desafios na atenção primária são vistos $^{(8)}$. Associado a isso, a promoção da saúde tem buscado acompanhar as alterações epidemiológicas ao consolidar e ampliar os serviços de proteção e recuperação da saúde por meio de planos de investigação e delineamento das demandas dos usuários antes mesmo que qualquer doença se instale ${ }^{(8)}$.

Como um fator de causa de morte e de demanda de atendimento no SUS, a queda é um importante evento multifatorial causador de agravo à saúde que necessita da criação de estratégias de intervenção e prevenção de novos casos. $O$ atendimento interdisciplinar em questão exige explorar o período anterior à queda do idoso e analisar os fatores intrínsecos e extrínsecos, a presença de morbidades, além de verificar as consequências pós-queda e sua influência na vida do idoso, a fim de reduzir seu tempo de recuperação, hospitalização e institucionalização(9).

As diretrizes do sistema de saúde e as medidas de crescimento de subsídios para garantir assistência ao idoso dependerão da condição clínica em que chegou à unidade de atendimento. Estratégias de atenção e melhoria da assistência são os desafios a que as políticas públicas de ações em promoção de saúde do idoso estão sujeitas ${ }^{(10)}$.

A promoção de saúde e os níveis de atenção à saúde devem ser atrelados às garantias previstas na Constituição Federal ${ }^{(11)}$ e no Estatuto do Idoso ${ }^{(12)}$, destinadas à preservação e promoção da integridade da pessoa idosa e à seguridade de seus direitos sociais, como um salário mínimo que possibilite condições de vida, acesso a programas de amparo e aos serviços de saúde locais de prevenção e manutenção, além de atendimento geriátrico e gerontológico em ambulatórios e na unidade de referência.

As políticas públicas de saúde voltadas para o acompanhamento do envelhecimento dos brasileiros, como as práticas educacionais e de promoção, necessitam de estudos que apresentem desfechos direcionados para a necessidade dos idosos, permitindo a execução de metodologias que auxiliam e ampliam o atendimento nas unidades de saúde ${ }^{(13)}$.

Assim, o objetivo do presente estudo consistiu em apresentar os aspectos socioeconômicos e a ocorrência de eventos de queda entre idosos atendidos no sistema municipal de saúde. 


\section{MÉTODOS}

Estudo descritivo e transversal realizado no município de Ituiutaba, Minas Gerais, Brasil, entre os meses de abril e junho de 2018, composto por 154 idosos, de ambos os sexos (feminino, $n=88$; masculino, $n=66$ ), com idade igual ou superior a 60 anos, não institucionalizados e atendidos nas unidades de saúde localizadas nos bairros Alvorada, Camargo, Elândia, Independência, Jardim do Rosário, Natal, Novo Horizonte, Novo Tempo 2, Pirapitinga, Santa Maria, Setor Norte e Sol Nascente.

Definiu-se o tamanho da amostra considerando o erro de 0,05 e erro amostral entre 4 e $0,8 \%$ para prevalências entre 50 e $1 \%$, respectivamente. O nível de confiança considerado é de $95 \%$ a partir da prevalência de idosos em Ituiutaba ${ }^{(14)}$ que está localizada na mesorregião do Pontal do Triângulo Mineiro e corresponde a $10 \%$ da população de Minas Gerais. Com 97.171 mil habitantes majoritariamente urbanos (95,8\%; n=93.122), dos quais 10.173 têm 60 anos ou mais, o município apresenta uma média de 2,2 salários mínimos mensais por trabalhador formal e tem índice de desenvolvimento humano de 0,739 ${ }^{(15)}$.

Realizou-se a coleta dos dados a partir da aplicação de questionário semiestruturado ${ }^{(16)}$ em entrevista conduzida pelos membros do Grupo de pesquisa de atenção preventiva e educativa em saúde do idoso da Universidade Federal de Uberlândia, campus Pontal. O instrumento contém perguntas que investigam questões socioeconômicas (origem rural ou urbana, idade, sexo, escolaridade, ocupação e renda familiar) e a sua condição clínica (manifestação de doenças crônicas e eventos de queda).

Após serem autorizados pelo Secretário Municipal de Saúde e pelo responsável da Unidade de Saúde, os pesquisadores se dividiram por ala ou sala de espera em direção a aqueles que apresentavam 60 anos ou mais. Os idosos eram convidados a participar da pesquisa enquanto aguardavam atendimento. Após a identificação dos pesquisadores, apresentação do estudo e aceite do participante, a entrevista era conduzida seguindo o instrumento de coleta.

Ocorrendo a saturação do grupo populacional no dia, finalizava-se a aplicação dos questionários. Consideraramse como critérios de inclusão idosos que pertenciam à população, com interesse em participar da entrevista e que concordaram em assinar o Termo de Consentimento Livre e Esclarecido; enquanto excluíram-se do estudo os idosos com incapacidade de responder ao questionário.

Tabularam-se os dados em planilha, com a posterior análise das variáveis por estatística descritiva por meio do programa BioEstat, versão 5.0, considerando as frequências absoluta (n) e relativa (\%) com base no número amostral.

A pesquisa obteve aprovação do Comitê de Ética da Universidade Federal de Uberlândia (UFU), Parecer $n$. $^{\circ}$ 3.070.463, e da Universidade Federal de Juiz de Fora, com coparticipação da UFU, Parecer n. ${ }^{\circ}$ 1.089.051.

\section{RESULTADOS}

A população entrevistada constituiu-se de 154 idosos, da qual $57,1 \%(n=88)$ era de mulheres, com maior prevalência desse sexo para a origem rural $(77,3 \% ; n=68)$ conforme descrito na Tabela I. A maioria das mulheres entrevistadas de origem rural apresentou idade entre 60 e 79 anos $(95,6 \% ; n=65)$ e dentre os homens advindos da zona rural observou-se predominância na faixa etária ente 70 e 79 anos $(53,6 \%$; $=30)$.

Em relação à ocupação a Tabela I aponta que, entre os aposentados e/ou pensionistas $(85,7 \% ; n=132), 80,7 \%$ do sexo feminino $(n=71)$ e $92,4 \%$ do sexo masculino $(n=61)$ declararam receber o benefício. Questionados sobre a renda, $85,1 \%(n=131)$ dos idosos afirmaram receber até 2 salários para ambas as origens (rural ou urbana) sendo o salário estimado em 954 reais naquele ano. Daqueles que revelaram ter renda superior a 2 salários $(14,3 \% ; n=22)$, $86,4 \%(n=19)$ corresponderam a idosos aposentados/pensionistas (Tabela I).

Entre os entrevistados, três idosos de origem rural não responderam às variáveis sobre ocupação (um homem e uma mulher) e renda familiar mensal (uma mulher).

O estudo demonstrou que a situação conjugal dos idosos atendidos nas unidades de saúde do município não apresentou variação relacionada à presença/ausência de companheiro(a) se comparada a origem do idoso. No entanto, ao analisar quanto ao sexo, $54,5 \%(n=48)$ das idosas mulheres declararam não possuir companheiro, em contrapartida de 40,9\% ( $\mathrm{n}=27$ ) dos idosos homens (Tabela I).

Quanto ao nível de escolaridade, do total dos idosos entrevistados $47,4 \%(n=73)$ declararam ter estudado menos de 4 anos. Ao avaliar a origem, os idosos advindos da zona rural corresponderam a $82,3 \%$ ( $n=102)$ com menor escolarização, ou seja, analfabetos, analfabetos funcionais ou que estudaram menos de 4 anos. As idosas que tiveram menos de quatro anos de escolarização corresponderam a $43,2 \%(n=38)$, em comparação aos homens com predominância de 53,0\% ( $n=35)$ para o esse mesmo grau de escolaridade, conforme descrito na Tabela I. 
Tabela I - Características sociodemográficas dos idosos atendidos nas unidades de saúde estratificadas por origem (urbana ou rural) e sexo, expressas em n (\%). Ituiutaba, Minas Gerais, Brasil, 2018.

\begin{tabular}{|c|c|c|c|c|c|c|c|}
\hline \multirow{3}{*}{ Variáveis } & \multicolumn{3}{|c|}{ Sexo Feminino } & \multicolumn{3}{|c|}{ Sexo Masculino } & \multirow{3}{*}{$\begin{array}{c}\begin{array}{c}\text { Total } \\
\mathrm{n}=154\end{array} \\
\mathrm{n}(\%)\end{array}$} \\
\hline & $\begin{array}{c}\text { Urbano } \\
n=20\end{array}$ & $\begin{array}{l}\text { Rural } \\
n=68\end{array}$ & $\begin{array}{l}\text { Total } \\
n=88\end{array}$ & $\begin{array}{c}\text { Urbano } \\
n=10\end{array}$ & $\begin{array}{l}\text { Rural } \\
n=56\end{array}$ & $\begin{array}{l}\text { Total } \\
n=66\end{array}$ & \\
\hline & n (\%) & n (\%) & n (\%) & n (\%) & n (\%) & n (\%) & \\
\hline \multicolumn{8}{|l|}{ Idade } \\
\hline 60 a 69 & $9(45,0)$ & $33(48,5)$ & $42(47,7)$ & $7(70,0)$ & $16(28,6)$ & $23(34,8)$ & $65(42,2)$ \\
\hline 70 a 79 & $10(50,0)$ & $32(47,1)$ & $42(47,7)$ & $3(30,0)$ & $30(53,6)$ & $33(50,0)$ & $75(48,7)$ \\
\hline$\geq 80$ & $1(5,0)$ & $3(4,4)$ & $4(4,6)$ & $0(0)$ & $10(17,9)$ & $10(15,2)$ & $14(9,1)$ \\
\hline \multicolumn{8}{|l|}{ Ocupação } \\
\hline Aposentado e/ou pensionista & $14(70,0)$ & $57(83,8)$ & $71(80,7)$ & $9(90,0)$ & $52(92,9)$ & $61(92,4)$ & $132(85,7)$ \\
\hline Sem ocupação & $1(5,0)$ & $5(7,4)$ & $6(6,8)$ & $0(0)$ & $0(0)$ & $0(0)$ & $6(3,9)$ \\
\hline Outra ocupação & $5(25,0)$ & $5(7,4)$ & $10(11,4)$ & $1(10,0)$ & $3(5,4)$ & $4(6,1)$ & $14(9,1)$ \\
\hline Não responderam & $0(0)$ & $1(1,4)$ & $1(1,1)$ & $0(0)$ & $1(1,8)$ & $1(1,5)$ & $2(1,3)$ \\
\hline \multicolumn{8}{|c|}{ Renda familiar mensal $(R \$ 954,00)$} \\
\hline 1 salário mínimo & $9(45,0)$ & $34(50,0)$ & $43(48,9)$ & $2(20,0)$ & $24(42,9)$ & $26(39,4)$ & $69(44,8)$ \\
\hline 1 a 2 salários & $10(50,0)$ & $26(38,2)$ & $36(40,9)$ & $4(40,0)$ & $22(39,3)$ & $26(39,4)$ & $62(40,3)$ \\
\hline Mais de 2 salários & $1(5,0)$ & $7(10,3)$ & $8(9,1)$ & $4(40,0)$ & $10(17,9)$ & $14(21,2)$ & $22(14,3)$ \\
\hline Não responderam & $0(0)$ & $1(1,5)$ & $1(1,1)$ & $0(0)$ & $0(0)$ & $0(0)$ & $1(0,6)$ \\
\hline \multicolumn{8}{|l|}{ Situação conjugal } \\
\hline Com companheiro & $9(45,0)$ & $31(45,6)$ & $40(45,5)$ & $5(50,0)$ & $34(60,7)$ & $39(59,1)$ & $79(51,3)$ \\
\hline Sem companheiro & $11(55,0)$ & $37(54,4)$ & $48(54,5)$ & $5(50,0)$ & $22(39,3)$ & $27(40,9)$ & $75(48,7)$ \\
\hline \multicolumn{8}{|l|}{ Escolaridade } \\
\hline Analfabeto & $2(10,0)$ & $12(17,6)$ & $14(15,9)$ & $1(10,0)$ & $4(7,1)$ & $5(7,6)$ & $19(12,3)$ \\
\hline Analfabeto funcional & $3(15,0)$ & $13(19,1)$ & $16(18,2)$ & $2(20,0)$ & $9(16,1)$ & $11(16,7)$ & $27(17,6)$ \\
\hline$<4$ anos & $8(40,0)$ & $30(44,1)$ & $38(43,2)$ & $1(10,0)$ & $34(60,7)$ & $35(53,0)$ & $73(47,4)$ \\
\hline$\geq 4$ anos & $7(35,0)$ & $13(19,1)$ & $20(22,7)$ & $6(60,0)$ & $9(16,1)$ & $15(22,7)$ & $35(22,7)$ \\
\hline
\end{tabular}

Sobre as quedas e seus aspectos relacionados, $37,7 \%(n=58)$ dos idosos afirmaram ter sofrido algum evento nos últimos 12 meses, apresentando prevalência entre aqueles com idade entre 70 e 79 anos $(55,2 \%$; $n=32)$ e do sexo feminino $(67,2 \%$; $n=39)$. Idosos com fratura em decorrência de queda corresponderam a 32,7\%, $(n=19)$, com predomínio entre mulheres $(57,9 \% ; n=11)$, como dispõe a Tabela II.

Do total de idosos que sofreram quedas, $22,4 \%(n=13)$ relataram apresentar alguma complicação pós-queda e $41,4 \%(n=24)$ declararam que há risco aparente para queda em sua casa, incluindo escadas, degraus e piso escorregadio, sendo maior entre as mulheres $(66,7 \% ; n=16)$ em decorrência da queda $(32,7 \% ; n=19)$, a chance de apresentarem fratura. (Tabela II).

Quanto às deficiências/distúrbios autorreferidos pelos idosos que sofreram queda nos últimos 12 meses, $65,5 \%$ $(n=38)$ afirmaram apresentar problemas relacionados à visão, 53,4\% $(n=31)$ declararam ter alguma doença no sistema osteomuscular, 34,5\% ( $n=34)$ relataram ter dificuldade para se locomover e $29,3 \%(n=17)$ dos idosos autorreferiram algum transtorno relacionado à saúde mental. Apenas $5,2 \%(n=3)$ dos idosos relataram apresentar alguma doença no sistema respiratório, sendo mais frequente entre as mulheres em todas as condições supramencionadas. (Tabela II). 
Tabela II - Variáveis relacionadas às quedas e deficiências/distúrbios autorreferidos de idosos atendidos nas unidades de saúde, estratificadas por sexo e expressas em n (\%). Ituiutaba, Minas Gerais, Brasil, 2018.

\begin{tabular}{|c|c|c|c|}
\hline Variáveis & Feminino $(n=39)$ & Masculino $(n=19)$ & Total $(n=58)$ \\
\hline \multicolumn{4}{|l|}{ Idade } \\
\hline 60 a 69 & $16(76,2)$ & $5(23,8)$ & $21(36,2)$ \\
\hline 70 a 79 & $20(62,5)$ & $12(37,5)$ & $32(55,2)$ \\
\hline$\geq 80$ & $3(60,0)$ & $2(40,0)$ & $5(8,6)$ \\
\hline Fratura & $11(57,9)$ & $8(42,1)$ & $19(32,7)$ \\
\hline Prótese & $2(66,6)$ & $1(33,3)$ & $3(5,2)$ \\
\hline Pós-queda & $8(61,5)$ & $5(38,5)$ & $13(22,4)$ \\
\hline Casa oferece risco & $16(66,7)$ & $8(33,3)$ & $24(41,4)$ \\
\hline \multicolumn{4}{|c|}{ Deficiência/distúrbio autorreferida(o) } \\
\hline Visual & $28(73,7)$ & $10(26,3)$ & $38(65,5)$ \\
\hline Osteomuscular & $23(74,2)$ & $8(25,8)$ & $31(53,4)$ \\
\hline Mobilidade & $14(70,0)$ & $6(30,0)$ & $20(34,5)$ \\
\hline Mental & $14(82,4)$ & $3(17,6)$ & $17(29,3)$ \\
\hline Respiratório & $2(66,6)$ & $1(33,3)$ & $3(5,2)$ \\
\hline
\end{tabular}

\section{DISCUSSÃO}

A mudança na demografia da população de um país está sincronizada com o comportamento das taxas de crescimento populacional e a velocidade de redistribuição das diferentes faixas etárias ${ }^{(17)}$.

Os resultados obtidos na população entrevistada revelam, nas unidades de saúde do município, um atendimento majoritariamente de idosos jovens, com idade entre 60 e 79 anos, mulheres e de origem rural. Contudo isso pode ser resultado de uma transição demográfica acentuada em meados de 1970, como apontado em registros do Instituto Brasileiro de Geografia e Estatística (IBGE) ${ }^{(18)}$.

A transformação demográfica e na estrutura populacional do país está atrelada ao estilo de vida da sociedade e a aspectos econômicos, comportamentais, culturais e, principalmente, sociais. Para acompanhar o aumento da participação desse grupo etário, políticas públicas voltadas para a redução de incapacidades e vulnerabilidades são cruciais para a manutenção do envelhecimento ativo ${ }^{(19)}$.

A prevalência do sexo feminino no presente estudo é justificada pelo levantamento do IBGE ${ }^{(15)}$, o qual revelou haver $53,6 \%$ de idosas neste município e $55,5 \%$ no Brasil. Esse achado reflete como o homem idoso culturalmente não apresenta o costume do autocuidado e não busca serviços de saúde que favorecem a senescência com qualidade, por isso medidas de atenção à saúde voltadas para o impacto do envelhecimento do homem precisam ser promovidas por meio de projetos e atividades na saúde básica ${ }^{(20)}$.

Quanto aos aspectos sociais, no presente estudo, a velhice feminina é marcada pelos piores indicadores quanto à renda (até 2 salários mínimos), escolaridade (menos de 4 anos), situação conjugal (sem companheiro) e ocupação (aposentadas e/ou pensionistas). Do total das mulheres entrevistadas, $89,8 \%(n=79)$ afirmaram receber até 2 salários mínimos no primeiro semestre de 2018, enquanto que, em 2015, observou-se a mesma renda para $69,7 \%$ das idosas, revelando crescimento da vulnerabilidade no município ${ }^{(14)}$.

Em situações de exercício de função remunerada, as mulheres historicamente sofreram com a desvalorização salarial, e hoje recebem aposentadoria abaixo dos valores pagos aos homens em decorrência da contribuição e/ou renda menor ${ }^{(21)}$. Comparando os dados epidemiológicos de 2018 com os obtidos em 2015 no município de Ituiutaba, Minas Gerais ${ }^{(22)}$, observa-se aumento no número de idosos aposentados e/ou pensionistas $(+13,3 \%)$ e diminuição da renda mensal familiar acima de 2 salários mínimos $(-2,9 \%)$.

O analfabetismo é um fator determinante na vida do idoso ao interferir na vulnerabilidade familiar. A baixa escolaridade está associada a outros fatores sociais, como baixos índices de renda per capita e instabilidade empregatícia, o que leva a pessoa idosa a apresentar dificuldade de acesso ao trabalho por não ter mão de obra especializada ${ }^{(23)}$. A condição de baixa escolaridade feminina no atual estudo, realizado na referida cidade mineira, 
apresentou uma proporção expressiva de entrevistadas com menos de quatro anos de escolarização ou que eram analfabetas e/ou analfabetas funcionais, revelando a necessidade de se incentivar a participação dos idosos em programas voltados para a aprendizagem e alfabetização.

A prevalência de idosas que não apresentam companheiro é uma realidade neste estudo, sendo a maioria de origem urbana. Um levantamento realizado em Viçosa, Minas Gerais, identificou a predominância de mulheres viúvas, solteiras e divorciadas $(62,5 \%)^{(24)}$.

Há uma íntima relação de variáveis sociais e econômicas com a ocorrência de quedas nesse grupo populacional, inclusive relacionando a melhor escolarização, vida ativa e renda com o menor risco de quedas e melhores condições de moradia e de acesso aos serviços de saúde ${ }^{(25)}$. Diante disso, e baseado no princípio da universalidade do SUS, é vista a necessidade de garantir a atenção básica para a população vulnerável do município, a qual não tem acesso aos serviços de saúde ${ }^{(11)}$.

Como o envelhecimento biológico é um processo irreversível, é também marcado pela perda da capacidade funcional decorrentes de alterações fisiológicas multifatoriais, como declínio osteomuscular, visual, auditivo, de mobilidade e cognitivo, e pode ser associado às doenças crônicas. Portanto, o acúmulo dessas alterações orgânicas potencializa os riscos de queda para a pessoa idosa, passando a ser uma preocupação das ações preventivas na atenção primária ${ }^{(26)}$.

Em Ituiutaba, cerca de um terço dos idosos afirmou ter sofrido algum evento de queda nos últimos 12 meses, tendo a maioria entre 70 e 79 anos. Outros estudos evidenciam a presença de eventos de queda, como visto em Pelotas, Rio Grande do Sul, com $28,1 \%{ }^{(27)}$, em Cuiabá, Mato Grosso, com $37,5 \%^{(28)}$, e em Curitiba, Paraná, com $34,6 \%{ }^{(29)}$.

Entre as várias condições autorreferidas pelas pessoas idosas que sofreram queda, os distúrbios/deficiências são as mais diretamente associadas, incluindo visual e osteomuscular como as mais citadas pelos idosos do estudo, seguidas de mobilidade e mental. O processo de senescência é seguido de alterações sensoriais, como o déficit visual, e o idoso pode apresentar incapacidades na execução de atividades, já que, com o avançar da idade cronológica os olhos apresentam perda de acuidade visual, diminuição do campo de visão e redução de noções de profundidade ${ }^{(30)}$.

No envelhecimento a pessoa idosa apresenta dificuldade em realizar suas atividades de vida diária (AVDs) devido à sarcopenia e osteopenia acentuadas, podendo perder cerca de $45 \%$ da força muscular até os 80 anos ${ }^{(31)}$. Estudo revela que $35 \%$ dos idosos precisam de auxílio para realizar suas AVDs e as atividades instrumentais de vida diária ${ }^{(27)}$. Esse dado endossa a teoria de que as mudanças estruturais influenciam na mobilidade e na capacidade funcional da pessoa idosa, resultando no aumento do número de quedas ${ }^{(32)}$.

A perda da capacidade funcional acentuada é vista nos idosos em situação de vulnerabilidade social, pois quanto maiores os indicadores de baixa escolaridade e baixa renda, maior será o seu isolamento, devido à dificuldade do idoso na sua autopercepção clínica e ao distanciamento dos centros urbanos, dificultando o acesso aos serviços de saúde(3).

O acesso aos serviços de saúde integral e assistência à pessoa idosa estão assegurados pela Constituição Federal ${ }^{(11)}$ e pelo Estatuto do Idoso ${ }^{(12)}$. Para atender os longevos distantes dos serviços públicos em saúde, ações voltadas para preservação da sua saúde física e mental podem ser realizadas no município seguindo o princípio da universalidade, por meio do atendimento individualizado e interdisciplinar, garantindo o suporte ao idoso.

Em uma metanálise realizada até o ano de 2013, verificou-se que a incapacidade funcional é variável entre os

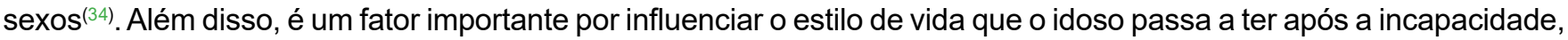
como a perda da qualidade de vida, maior dependência dos familiares e exposição a situações de agravamentos à saúde, como quedas, fraturas, institucionalização e até a morte(32).

As fraturas mostraram ser a consequência pós-queda mais relatada pelos idosos do presente estudo. Os eventos de queda que resultam em fraturas apresentam um aumento expressivo na morbidade e mortalidade, além de terem um aspecto negativo na mobilidade do idoso ${ }^{(35)}$. As políticas de promoção da saúde socioeducativas, voltadas para a manutenção da vida através da prevenção e rastreamento de doenças, bem como as de acesso aos serviços de saúde multidisciplinares, são essenciais na melhor idade para a redução dos riscos de queda ${ }^{(36)}$.

É importante avaliar não só os fatores intrínsecos durante a senescência, mas também as causas ambientais e comportamentais que aumentam os riscos de queda entre idosos ${ }^{(36)}$. Na presente pesquisa, verificou-se um alto percentual de relatos sobre o risco presente em suas residências favorecendo eventos de queda entre os idosos entrevistados, como também revelado em estudo realizado em Pelotas, Rio Grande do Sul(28), no qual 56,4\% dos idosos caidores sofreram quedas dentro da sua própria casa. 
Um estudo multicêntrico realizado com pacientes de seis hospitais de ortogeriatria de Barcelona, Espanha, identificou que a maioria das causas de queda é por causa de fatores intrínsecos $(78,5 \%)$ ou pela combinação dos dois fatores $(20,6 \%)^{(37)}$. Assim, planejar estratégias de prevenção por meio do atendimento multidisciplinar voltado para a orientação e educação do idoso é uma medida fundamental para que o SUS tenha atenção à saúde de maneira integral, com a responsabilidade de garantir o bem-estar e o cuidado no envelhecimento ${ }^{(38)}$.

Desse modo, delineamentos epidemiológicos que buscam traçar o perfil de uma população e a ocorrência de doenças nela, como realizado neste estudo, são fundamentais para nortear as políticas públicas em saúde baseadas na promoção da qualidade de vida e na prevenção de fatores de causa de morbidades. Além disso, são importantes para direcionar as ações de educação em saúde na população de risco, com a finalidade de intervir antes do acometimento da doença( ${ }^{(2)}$.

As limitações desta investigação devem ser ressaltadas, uma vez que há a possibilidade de alguns vieses de desfecho por parte dos entrevistados, como viés de esquecimento quando questionados sobre quedas e consequências pós-quedas, viés de omissão de renda e/ou ocupação. Os dados interpretam a situação de vida de idosos atendidos nas unidades de atenção básica de Ituiutaba, portanto não devem ser generalizados para a população, mas podem ajudar a repensar a promoção de saúde e a segurança contra quedas dessa população.

\section{CONCLUSÃO}

Os eventos de queda autorreferidos pelos idosos investigados associam-se aos fatores inerentes do envelhecimento biológico, com acentuada feminização, aposentadas e/ou pensionistas, com baixa renda e escolaridade, mas também aos fatores extrínsecos associados ao ambiente onde vivem.

\section{CONFLITOS DE INTERESSE}

Não houve conflitos de interesses na execução desta pesquisa.

\section{CONTRIBUIÇÕES}

Alexandre Azenha Alves de Rezende contribuiu com a elaboração e delineamento do estudo; e a redação e/ ou a revisão do manuscrito. Ériks Oliveira Silva e Luciana Karen Calábria contribuíram com a elaboração e delineamento do estudo; a aquisição, análise e interpretação dos dados; e a redação e/ou a revisão do manuscrito.

\section{REFERÊNCIAS}

1. Ramos JS, Carvalho FSS Filha, Silva RNA. Avaliação da adesão ao tratamento por idosos cadastrados no programa do HiperDia. Rev Gest Sist Saúde. 2015;4(1):29-39.

2. Instituto Brasileiro de Geografia e Estatística. Tábua completa de mortalidade para o Brasil: breve análise da evolução da mortalidade no Brasil [Internet]. 2018 [acesso em 2019 Maio 13]. Disponível em: https:// biblioteca.ibge.gov.br/visualizacao/livros/liv101628.pdf

3. Instituto Brasileiro de Geografia e Estatística. Pesquisa Nacional por Amostra de Domicílios Contínua: características gerais dos moradores 2012-2016 [Internet]. 2018 [acesso em 2019 Maio 13]. Disponível em: https://www.ibge.gov.br/estatisticas/sociais/trabalho/17270-pnad-continua.html?=\&t=downloads

4. Toleto DR, Barela JA. Diferenças sensoriais e motoras entre jovens e idosos: contribuição somatossensorial no controle postural. Rev Bras Fisioter. 2010;14(3):267-75.

5. Ministério da Saúde (BR). Agência Nacional de Saúde Suplementar. Plano de cuidado para idosos na saúde suplementar [Internet]. Brasília: MS; 2012 [acesso em 2019 Out 03]. Disponível em: http://www.ans.gov.br/ images/stories/Materiais_para_pesquisa/Materiais_por_assunto/20121004_plano_cuidado_idosos.pdf

6. Abreu DROM, Novaes ES, Oliveira RR, Mathias TAF, Marcon SS. Internação e mortalidade por quedas em idosos no Brasil: análise de tendência. Ciênc Saúde Colet. 2016;3(4):131-41.

7. Pinho TAM, Silva AO, Tura LFR, Moreira MASP, Gurgel SN, Smith AAF, et al. Avaliação do risco de quedas em idosos atendidos em Unidade Básica de Saúde. Rev Esc Enferm USP. 2012;46(2):320-27. 
8. Conselho Nacional de Secretários de Ministério da Saúde (BR). SUS 20 anos [internet]. 2009 [acesso em 2019 Out 03]. Disponível em: https://www.conass.org.br/bibliotecav3/pdfs/sus20anosfinal.pdf

9. Moraes SA, Soares WJS, Lustosa LP. Bilton TL, Ferrioli E, Perracini MR. Características das quedas em idosos que vivem na comunidade: estudo de base populacional. Rev Bras Geriatr Gerontol. 2017;20(5):693704.

10. Antunes JLFA, Chiavegatto ADP Filho, Duarte YAO, Lebrão ML. Desigualdades sociais na autoavaliação de saúde dos idosos da cidade de São Paulo-SP. Rev Bras Epidemiol. 2018;21(2):1-14.

11. Senado Federal (BR), Secretaria de Editoração e Publicações, Coordenação de Edições Técnicas. Constituição da República Federativa do Brasil: : texto constitucional promulgado em 5 de outubro de 1988, com as alterações determinadas pelas Emendas Constitucionais de Revisão nos 1 a 6/94, pelas Emendas Constitucionais nos 1/92 a 91/2016 e pelo Decreto Legislativo no 186/2008 [Internet]. Brasília: Senado Federal; 2016 [acesso em 2019 Set 12]. Disponível em: https://www2.senado.leg.br/bdsf/bitstream/handle/ id/518231/CF88_Livro_EC91_2016.pdf

12. Ministério da Saúde (BR). Estatuto do Idoso [Internet]. 2013 [acesso em 2019 Set 12]. Disponível em: http:// bvsms.saude.gov.br/bvs/publicacoes/estatuto_idoso_3edicao.pdf

13. Janini JP, Bessler $D$, Vargas $A B$. Educação em saúde e promoção da saúde: impacto na qualidade de vida do idoso. Saúde Debate. 2015;39(105):480-90.

14. Melo JV, Santos ALM, Rezende AAA, Calábria LK. Hábitos alimentares dos idosos atendidos nos Programas de Saúde da Família (PSF) em Ituiutaba-MG. Rev Med Saúde Brasília. 2017;6(2):154-66.

15. Instituto Brasileiro de Geografia e Estatística. Sinopse do censo demográfico de 2010 [Internet]. 2010 [acesso em 2019 Maio 13]. Disponível em: https://censo2010.ibge.gov.br/sinopse/index.php?dados=27\&uf=31

16. Faria LR, Calábria LK, Silva CLA, Albuquerque MCB, Santos RPE, Cau SBA. Atenção preventiva e educativa em saúde do idoso: uma proposta de integração de saberes e práticas. Estud Interdiscipl Envelhec. 2016;21(1);35-54.

17. Instituto Brasileiro de Geografia e Estatística. Mudanças demográficas no Brasil no início do século XXI [Internet]. 2015 [acesso em 2019 Set 12]. Disponível em: https://biblioteca.ibge.gov.br/visualizacao/livros/ liv93322.pdf

18. Instituto Brasileiro de Geografia e Estatística. Relações entre as alterações históricas na dinâmica demográfica brasileira e os impactos decorrentes [Internet]. 2016 [acesso em 2019 Maio 13]. Disponível em: https://biblioteca.ibge.gov.br/visualizacao/livros/liv98579.pdf

19. Organização das Nações Unidas. Relatório do Desenvolvimento Humano 2014. Sustentar o progresso humano: reduzir as vulnerabilidades e reforçar a resiliência [Internet]. 2014 [acesso em 2019 Set 13]. Disponível em: http://hdr.undp.org/sites/default/files/hdr2014_pt_web.pdf

20. Queiroz TS, Rehem TCMSB, Stival MM, Funghetto SS, Lima LR, Cardoso BG, et al. Como homens idoso cuidam de sua própria saúde na atenção básica? Rev Bras Enferm. 2017;71(1):599-606.

21. Belo I. Velhice e mulher: vulnerabilidades e conquistas. Rev Feminismos. 2013;1(3):1-20.

22. Faria MF, Rezende AAA, Calábria LK. Relação da depressão com aspectos sociodemográficos em idosos residentes nas zonas urbana e rural de Ituiutaba-MG. Rev Saúde Fís Ment. 2017;5(1):28-46.

23. Souza RA, Alvarenga MRM, Amendola F, Silva TMR, Yamashita $\mathrm{CH}$, Oliveira MAC. Vulnerabilidade de famílias de idosos assistidos pela Estratégia Saúde da Família. Rev Bras Enferm. 2015;68(2):244-52.

24. Almeida AV, Mafra SCT, Silva EP, Kanso S. A Feminização da velhice: em foco as características socioeconômicas, pessoais e familiares das idosas e o risco social. Textos e Contextos (Porto Alegre). 2015;14(1):115-31.

25. Pereira GN, Morsch P, Lopes DGC, Trevisan MD, Ribeiro A, Navarro JHN, et al. Fatores socioambientais associados à ocorrência de quedas em idosos. Ciênc Saúde Colet. 2013;18(12):3507-14.

26. Santos RKM, Maciel ACC, Britto HMJS, Lima JCC, Souza TO. Prevalência e fatores ao risco de quedas em idosos adscritos a uma Unidade Básica de Saúde do município de Natal, RN, Brasil. Ciênc Saúde Colet. 2015;20(12):3753-62. 
27. Vieira LS, Gomes AP, Bierhals IO, Farías-Antúnez S, Ribeiro CG, Miranda VIA, et al. Quedas em idosos no Sul do Brasil: prevalência e determinantes. Rev Saúde Pública. 2018;52(22):1-13.

28. Cruz DT, Ribeiro LC, Vieira MT, Teixeira MTB, Bastos RR, Leite ICG. Prevalência de quedas e fatores associados em idosos. Rev Saúde Pública. 2012;46(1):138-46.

29. Vaccari E, Lenardt MH, Willig MH, Betiolli SE, Andrade LAS. Segurança do paciente idoso e o evento no ambiente hospitalar. Cogitare Enferm. 2016;21(5):1-9.

30. Abreu HCA, Reiners AAO, Azevedo RCS, Silva AMC, Abreu DROM, Oliveira AD. Incidência e fatores preditores de quedas de idosos hospitalizados. Rev Saúde Pública. 2015;49(37):1-9.

31. Aveiro MC, Driusso P, Barham EJ, Pavarini SCI, Oishi J. Mobilidade e risco de quedas de população idosa da comunidade de São Carlos. Ciênc Saúde Colet. 2012;17(9):2481-8.

32. Farías-Antúnez S, Lima NP, Bierhal IO, Gomes AP, Vieira LS, Tomasi E. Incapacidade funcional para atividades básicas e instrumentais da vida diária: um estudo de base populacional com idosos de Pelotas, Rio Grande do Sul, 2014. Epidemiol Serv Saúde. 2018;27(2):1-14.

33. Barbosa BR, Almeida JM, Barbosa MR, Rossi-Barbosa LAR. Avaliação da capacidade funcional dos idosos e fatores associados à incapacidade. Ciênc Saúde Colet. 2013;19(8):3317-25.

34. Campos ACV, Almeida MHM, Campos GV, Bagutchi TF. Prevalência de incapacidade funcional por gênero em idosos brasileiros: uma revisão sistemática com metanálise. Rev Bras Geriatr Gerontol. 2016;19(3):54559.

35. Akyol Y, Ulus Y, Tander B, Tomak L, Zahiroğlu Y, Bilgici A, et al. Falls, fear of falling, and associated factors in ambulatory patients with rheumatoid arthritis: a comparative study with healthy control. Turk J Phys Med Rehabil. 2018;64(3):213-21.

36. Soares DS, Mello LM, Silva AS, Nunes AA. Análise dos fatores associados a quedas com fratura de fêmur em idosos; um estudo caso-controle. Rev Bras Geriatr Gerontol. 2015;18(2):238-48.

37. Formiga F, Chivite D, Ruiz D, Navarro M, Perez Castejon JM, Duaso E, et al. Clinical evidence of diabetes mellitus end-organ damage as risk factor for falls complicated by hip fracture: a multi-center study of 1225 patients. Diabetes Res Clin Pract. 2015;109(2):233-7.

38. Godoy AR, Adami FS. Estado nutricional e qualidade de vida de em adultos idosos com depressão. Rev Bras Promoç Saúde. 2019;32:1-12.

\author{
Endereço do primeiro autor: \\ Ériks Oliveira Silva \\ Universidade Federal de Uberlândia - UFU \\ Rua 20, 1600 \\ Bairro: Tupã \\ CEP: 38304-402 - Ituiutaba - MG - Brasil \\ E-mail: eriksoliveirabioufu@gmail.com

\section{Endereço para correspondência:} \\ Luciana Karen Calábria \\ Universidade Federal de Uberlândia - UFU \\ Rua 20, 1600 \\ Bairro: Tupã. \\ CEP: 38304-402 - Ituiutaba - MG - Brasil \\ E-mail: Ikcalabria@ufu.br
}

Como citar: Silva EO, Rezende AAA, Calábria LK. Aspectos socioeconômicos e eventos de queda entre idosos atendidos no sistema público de saúde. Rev Bras Promoç Saúde. 2019;32:9532. 